\title{
Optimizing cancer pain management in resource-limited settings
}

\author{
Sam H. Ahmedzai ${ }^{1}$ Mary Jocylyn Bautista ${ }^{2} \cdot$ Kamel Bouzid $^{3} \cdot$ Rachel Gibson $^{4} \cdot$ Yuddi Gumara $^{5}$. \\ Azza Adel Ibrahim Hassan ${ }^{6,7}$. Seiji Hattori ${ }^{8}$. Dorothy Keefe ${ }^{9}$. Durval Campos Kraychete ${ }^{10}$. Dae Ho Lee ${ }^{11}$. \\ Kazuo Tamura ${ }^{12}$. Jie Jun Wang ${ }^{13}$. CAncer Pain management in Resource-limited settings (CAPER) Working Group
}

Received: 22 March 2018 / Accepted: 10 September 2018 / Published online: 21 September 2018

(C) The Author(s) 2018

\begin{abstract}
Purpose Adequate cancer pain management (CPM) is challenging in resource-limited settings, where current international guideline recommendations are difficult to implement owing to constraints such as inadequate availability and accessibility of opioids, limited awareness of appropriate opioid use among patients and clinicians, and lack of guidance on how to translate the best evidence into clinical practice. The multinational and multidisciplinary CAncer Pain managEment in Resource-limited settings (CAPER) Working Group proposes a two-step initiative to bridge clinical practice gaps in CPM in resource-limited settings.

Methods A thorough review of the literature, a steering committee meeting in February 2017, and post-meeting teleconference discussions contributed to the development of this initiative. As a first step, we developed practical evidence-based CPM algorithms to support healthcare providers (HCPs) in tailoring treatment according to availability of and access to resources. The second part of the initiative proposes a framework to support an effective implementation of the CPM algorithms that includes an educational program, a pilot implementation, and an advocacy plan.

Results We developed CPM algorithms for first-line use, breakthrough cancer pain, opioid rotation, and refractory cancer pain based on the National Comprehensive Cancer Network guidelines and expert consensus. Our proposed educational program emphasizes the practical elements and illustrates how HCPs can provide optimal CPM according to evidence-based guidelines despite varied resource limitations. Pilot studies are proposed to demonstrate the effectiveness of the algorithms and the educational program, as well as for providing evidence to support a draft advocacy document, to lobby policymakers to improve availability and accessibility of analgesics in resource-limited settings.
\end{abstract}

Electronic supplementary material The online version of this article (https://doi.org/10.1007/s00520-018-4471-z) contains supplementary material, which is available to authorized users.

Jie Jun Wang

jiejunw19@163.com

1 National Institute of Health Research Clinical Research Network for Cancer, Leeds, UK

2 Benavides Cancer Institute, University of Santo Tomas Hospital, Manila, Philippines

3 Medical Oncology Department, Pierre \& Marie Curie Center, Algiers, Algeria

4 Division of Health Sciences, University of South Australia, Adelaide, Australia

5 National Cancer Center Dharmais Hospital, Jakarta, Indonesia

6 Supportive \& Palliative Care Section, Medical Oncology Department, National Center for Cancer Care and Research, Hamad Medical Corporation, Doha, Qatar
Cancer Management \& Research, Medical Research Institute, Alexandria University, Alexandria, Egypt

8 Department of Cancer Pain Management, The Cancer Institute Hospital of JFCR, Tokyo, Japan

9 Department of Medicine, Faculty of Health Sciences, University of Adelaide, Adelaide, Australia

10 College of Medicine, Federal University of Bahia, Salvador, Bahia, Brazil

11 Department of Oncology, University of Ulsan College of Medicine, Asan Medical Center, Seoul, Republic of Korea

12 General Medical Research Center, Fukuoka University School of Medicine, Fukuoka, Japan

13 Department of Oncology, Shanghai Changzheng Hospital, Second Military Medical University, No. 64 He Tian Road, Shanghai 200070, People's Republic of China 
Conclusions These practical evidence-informed algorithms and the implementation framework represent the first multinational step towards achieving optimal CPM in resource-limited settings.

Keywords Cancer pain $\cdot$ Analgesic $\cdot$ Resource-limited $\cdot$ Algorithm $\cdot$ Education $\cdot$ Implementation

\section{Introduction}

Pain is one of the most prevalent consequences of cancer affecting up to $70 \%$ of all patients with cancer over the course of their disease [1]. Despite several treatment options and best practice guidelines, undertreatment of cancer-related pain is widely reported; up to one-third of patients do not receive analgesia proportional to their pain intensity [2-4].

Adopting best practices in cancer pain management (CPM) is particularly challenging in resource-limited settings [5]. Inadequate availability of and access to opioid analgesics, the cornerstone for treating cancer pain, are also major challenges in resource-limited settings where many patients are first diagnosed with advanced-stage cancer [6, 7; Supplementary resource 1]. Global consumption of opioid analgesics is predominantly driven by high-income countries, whereas $75 \%$ of the world population, mainly in lower- and middle-income countries, lack adequate access to pain relief [8]. Impediments to opioid availability and accessibility in resource-limited settings include restrictive opioid formularies, over-regulation related to licensing and prescription, fear of opioid diversion to illicit channels, issues related to supply and distribution, cultural attitudes towards pain, patients and healthcare provider (HCP) concerns over addiction, and restricted financial resources [8-13].

Thus, there remains a significant gap between "optimal practice" and actual practice in CPM in resource-limited settings. While several international guidelines provide recommendations on managing cancer pain, these are often predicated on maximal resource availability and cannot be easily and appropriately implemented in areas of resource scarcity [14]. Clinical guidelines also provide little information and guidance on how to translate evidence-based recommendations into clinical practice [15]. Additionally, several diverse factors influence the implementation of best practice guidelines (Fig. 1) [16, 17].

Especially in palliative care for advanced disease, lack of education of HCPs, policymakers, and patients continues to be a key limiting factor contributing to inadequate CPM [10, 18-20]. This may manifest itself as misconceptions (including opiophobia), improper use of analgesics, and restrictive policies governing availability and accessibility of analgesics [9-13, 18, $21,22]$. Some programs have tried to address these educational needs on a national basis, such as the essential pain management

Fig. 1 Factors influencing the implementation of guidelines for cancer pain management

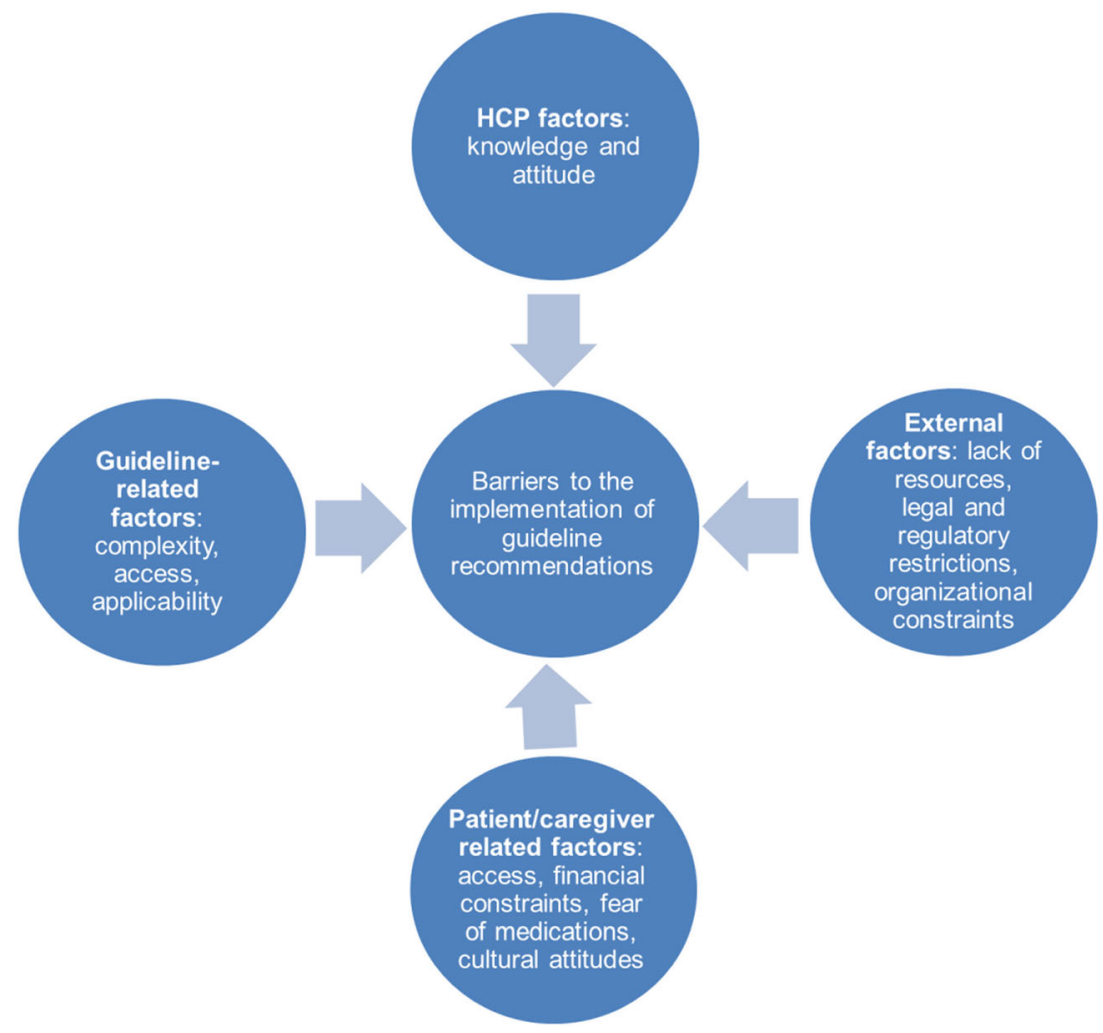


course (adapted with permission from the Faculty of Pain Medicine Australia and New Zealand College of Anaesthetists) in the Philippines and the Palliative care Emphasis program on symptom management and Assessment for Continuous medical Education (PEACE), a palliative care education program in Japan [23, 24].

As a step towards ensuring the provision of optimal CPM across all settings, a multinational consortium - the CAncer Pain managEment in Resource-limited settings (CAPER) Working Group (WG) - proposes a two-part, expert-driven initiative. We favor the use of the term resource-limited "settings" rather than "countries/regions" to underscore the heterogeneity of resource availability even within a country/ region. The ultimate goal of this initiative is to facilitate highquality care based on adaptations of evidence-based guidelines to improve pain-related clinical outcomes for all patients with cancer pain. This paper details the CAPER WG initiative emphasizing the collaborative process undertaken in providing physicians with (1) practical evidence-based algorithms for optimizing CPM amidst limited availability of analgesics and (2) a recommended implementation framework through education and advocacy that can be carried out by any groups or institutions to improve the availability and accessibility of opioids in resource-limited settings.

\section{Methods}

The CAPER WG - with a Steering Committee of eight members and an Advisory Group of four members - is comprised of a multidisciplinary consortium of oncologists, pain specialists and palliative care experts from Algeria, Australia, Brazil, China, Indonesia, Japan, the Philippines, Qatar, South Korea, and the UK [Supplementary resource 2].

At the outset, we reviewed the literature from January 2007 to December 2016 on the barriers to effective CPM across the Asia-Pacific, Latin America, and the Middle East and North Africa and efforts undertaken to overcome these challenges [refer to Supplementary resource 3 for a brief summary of the literature review]. Additionally, a survey completed by the working group members [Supplementary resource 4] provided further insights into current practices and challenges in CPM in resource-limited settings as well as in resource-rich countries. We further developed our initiative through telephonic discussions on the objectives and anticipated outcomes.

The CAPER Steering Committee subsequently convened at a meeting in Shanghai, China, in early February 2017, to discuss the challenges in CPM and review recommendations derived from existing published guidelines. While there are several national and international guidelines on CPM in adults, the Steering Committee focused on recommendations from the US National Comprehensive Cancer Network
(NCCN) [25], as it is most frequently referred to by a majority of the CAPER WG. Several topics were discussed in CPM including pain assessment, first-line treatment, treatment for breakthrough pain, subsequent-line treatment, adjuvant analgesics, management of opioid-related adverse events (AEs), psychosocial support, and patient and family/caregiver education. Acknowledging known constraints related to opioid availability and accessibility, the CAPER WG identified the adaptation of treatment-oriented guidelines to resourcelimited settings as the most relevant to providing optimal CPM. Therefore, the Steering Committee drafted the principal methodology for developing management algorithms to facilitate CPM by HCPs in settings with scarce resources (Fig. 2). The WG then amalgamated evidence-based recommendations and their own clinical experience into resource-sensitive algorithms that optimize standard of care for first-line CPM, breakthrough cancer pain (BTcP) management, opioid rotation, and refractory pain.

To support the effective translation of these algorithms into clinical practice, the Steering Committee also deliberated on the importance of education in achieving the aims of the initiative, ways to optimize the use of available analgesics, and strategies to advocate for improved availability and accessibility of opioids. This resulted in the preliminary drafts of an education and advocacy framework, which was further

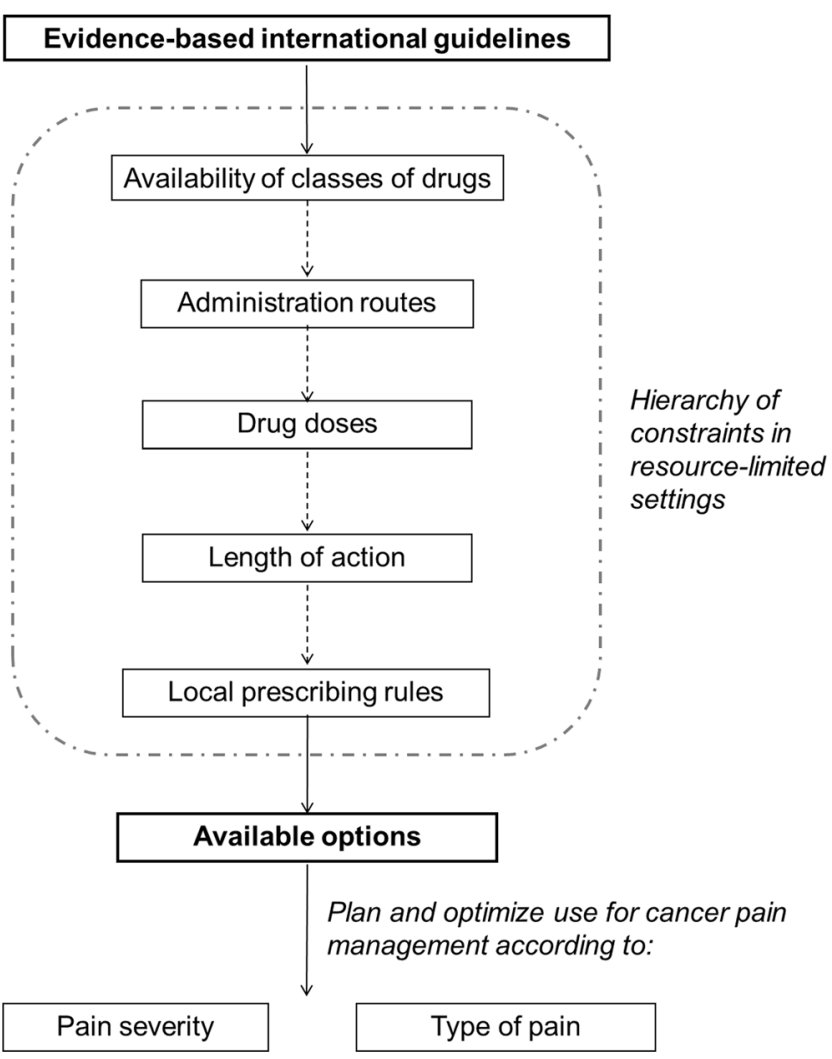

Fig. 2 Principal methodology to implement evidence-based international guidelines while optimizing the use of available analgesics 
expanded by the CAPER WG through multiple online discussions.

\section{Results}

To achieve the goal of our initiative, we have developed a structure that encompasses two main elements-(1) CPM management algorithms and (2) a proposed framework for an educational program and pilot implementation, also incorporating an advocacy plan [Supplementary resource 5].

\section{Cancer pain management algorithms}

Using a multimodal approach based on literature reviews and our clinical experience, we have developed four management algorithms, as a starting point for tackling some of the most problematic areas in CPM, to support optimal outcomes from using locally available analgesics: (1) first-line treatment of cancer pain (Fig. 3A), (2) management of BTcP (Fig. 3B), (3) opioid rotation in CPM (Fig. 3C), and (4) management of refractory cancer pain (Fig. 3D). We acknowledge that there are many more drug therapies in CPM, such as the use of non-opioid "adjuvant" medications, as well as non-pharmacological interventions (e.g., cancer-modifying therapies which include radiation, surgery, and interventional pain management), but the current algorithms represent the first stage.

For all these situations, opioid selection should be individualized based on availability, cost, patient characteristics and preference, and physician familiarity. As with all guidelines, clinicians must ultimately choose the optimal option based on a balance of evidence and practical considerations. While treatment should be initiated at the lowest effective dose, this dose must subsequently be personalized through gradual dose titration, adjusting dosing up or down to identify the optimal level that maximizes pain relief while minimizing AEs

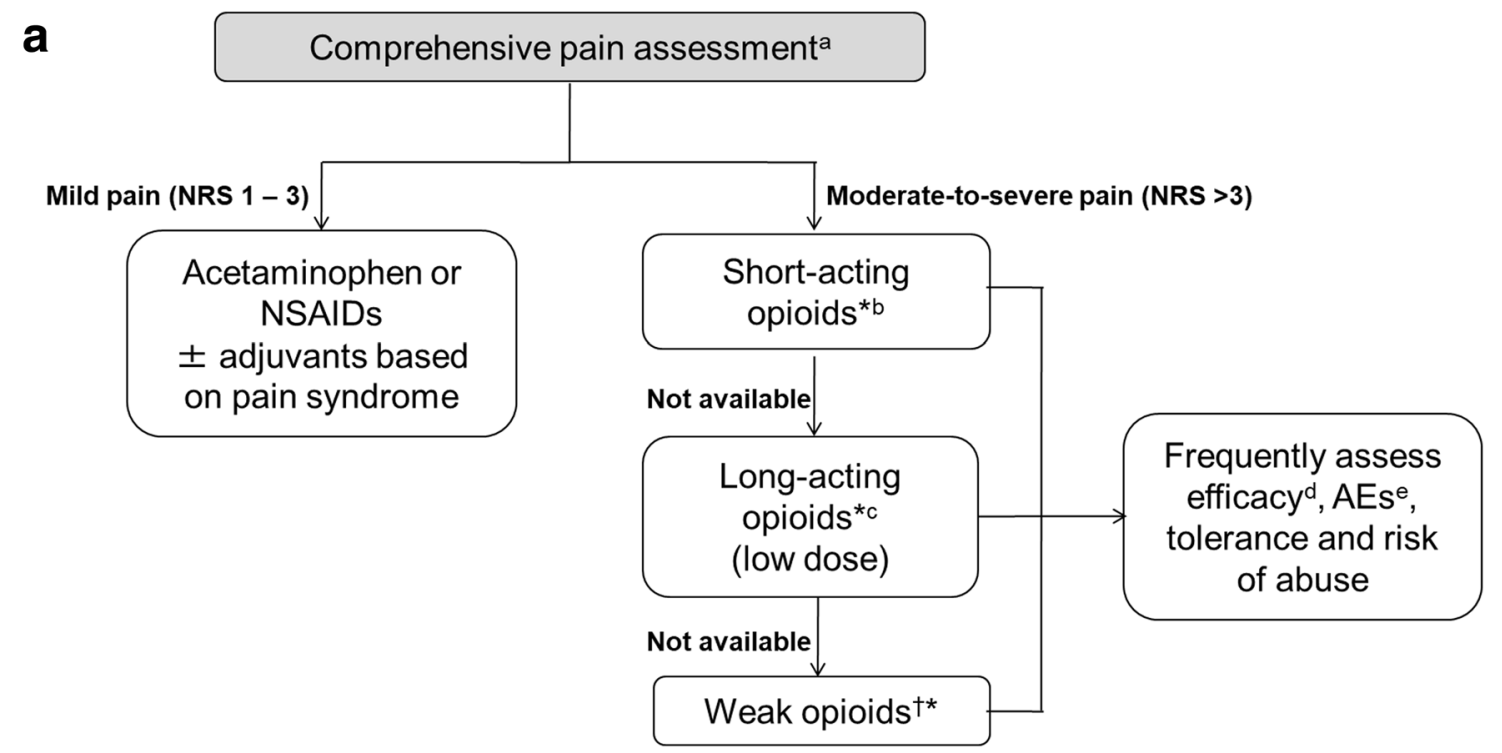

Fig. 3 A Algorithm for the first-line management of cancer pain in opioidnaïve patients. B Algorithm for the management of breakthrough cancer pain. C Algorithm for the management of opioid rotation in cancer pain management. D Algorithm for the management of refractory cancer pain. AEs, adverse events; NSAIDs, non-steroidal anti-inflammatory drugs; NRS, numerical rating scale; h, hour; NMDA, $N$-methyl D-aspartate 
b

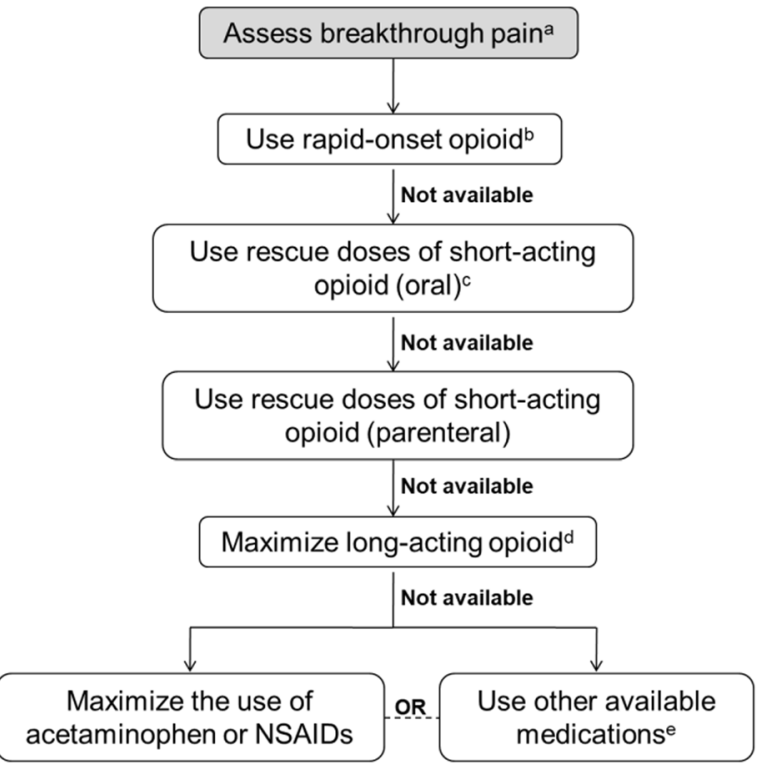

Re-assess pain, AEs, tolerance, and risk of abuse

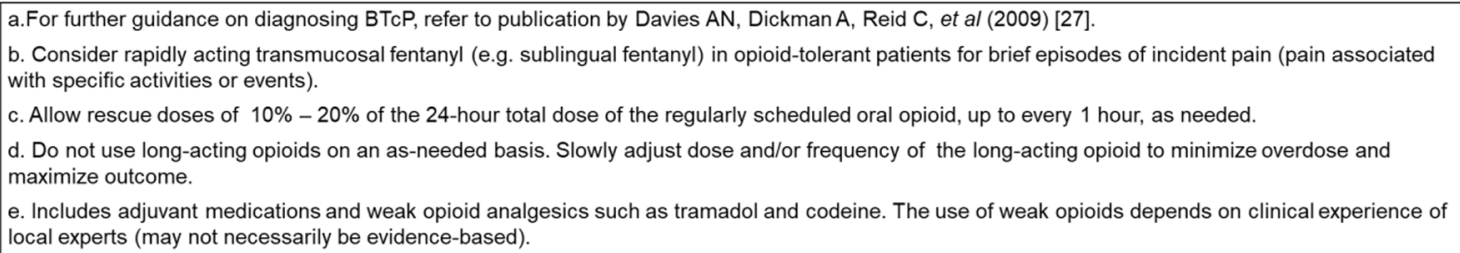

Fig. 3 (continued)

(Fig. 3). Management of BTcP should proceed in parallel with finding the correct dose of "around the clock" (ATC) pain control (Fig. 3B). If there is an unfavorable balance between analgesia and AEs in spite of several dose adjustments, clinicians should re-evaluate the therapeutic strategy and consider alternatives, such as more active and targeted management of AEs, addition of adjuvant analgesics, and opioid rotation (Fig. 3C). When pain persists despite these measures, clinicians should consider consulting with medical, radiation or interventional oncologists, surgeons, and palliative medicine or pain management specialists skilled in the use of specialized measures such as ketamine or palliative sedation (Fig. 3D).

Irrespective of resource availability, clinicians should conduct a thorough initial pain assessment, monitor patients frequently, and manage opioid-induced AEs [Supplementary resource 6]. Additionally, the multidimensional nature of cancer pain necessitates all HCPs to provide holistic psychosocial support and patient and caregiver education through a multiprofessional team.

\section{Proposed implementation framework}

To demonstrate the utility of the algorithms developed above, we propose a theoretical implementation framework that is based on strong evidence-based principles, includes essential elements in education and advocacy to ensure effective implementation, and can be utilized or adapted by any groups or institutions to improve CPM in their own settings based on their available resources. The implementation will need to be tested for feasibility and potential for clinically useful effectiveness in different settings. At the time of the submission of the manuscript, we are looking at putting the proposed framework into practice at a range of pilot implementation sites.

\section{Educational program}

The CAPER WG has determined an educational program that will be rolled out across resource-limited regions, with adaptations to suit different healthcare settings (Table 1). Intended for all HCPs involved in the care of patients with cancer, the educational program should include pertinent information on appropriate use of analgesics and guidance on how the CPM algorithms can be used clinically in different resource settings. An educational session in an interactive format of case study discussions and workshops will provide a practical element and allow participants to evaluate their understanding and apply their learning in real-life cases.

As part of the educational resource, a toolkit will be developed that can be customized to suit the varied learning needs of participants. It should contain the algorithms, the complete 


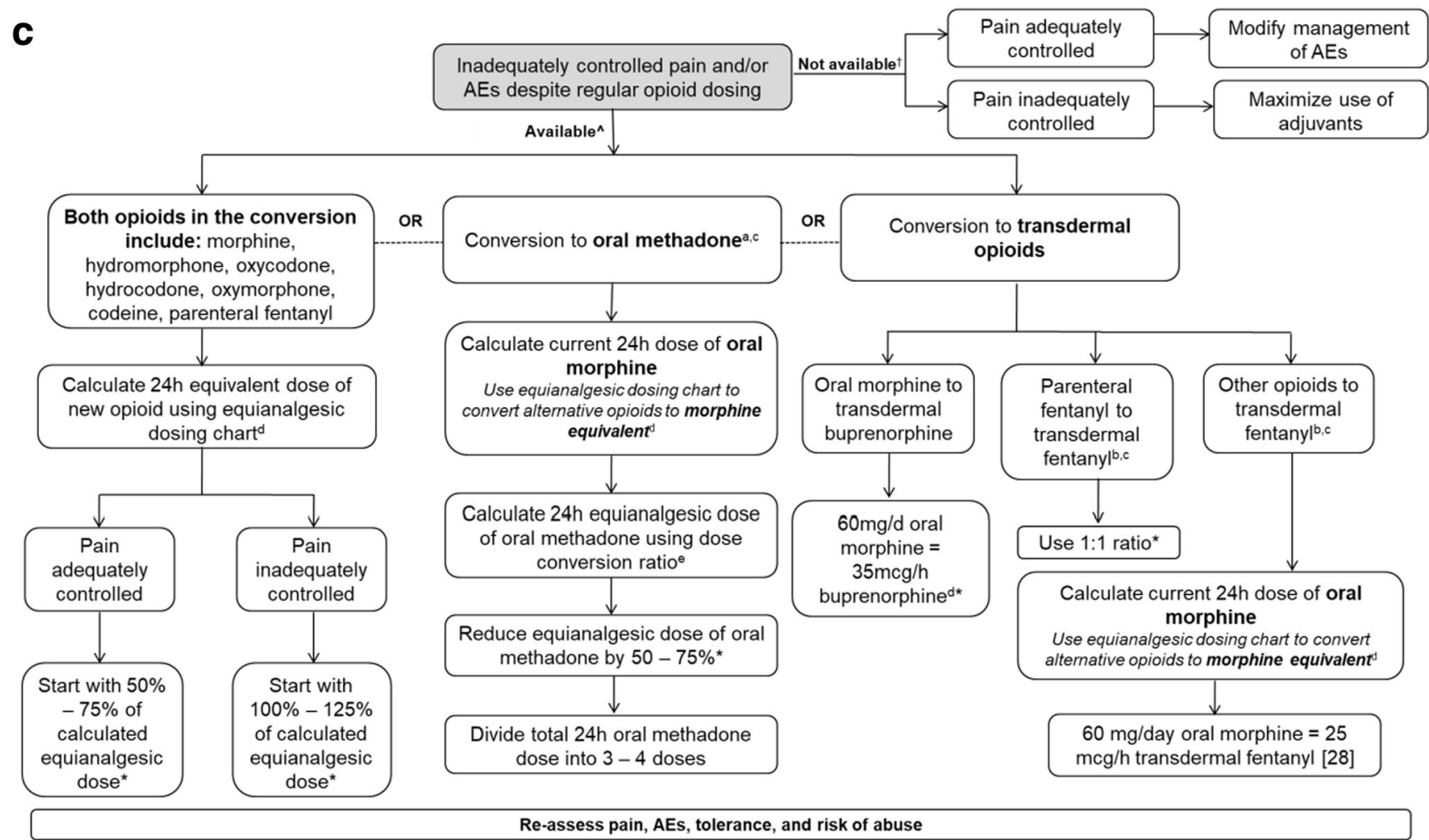

$\wedge$ Individualize drug selection based on clinical characteristics, prior experience, availability, cost, patient preference, and psychosocial considerations. No evidence-based dosing ratio for conversion between transmucosal fentanyl formulations or from transmucosal fentanyl to other opioids; see package insert for dosing information.

$\dagger$ Based on Expert Opinion; not necessarily evidence-based

* Titrate dose based on steady state levels, drug interactions, pain syndrome, and patient characteristics (age, renal and hepatic function, comorbidities).

a. Only recommended to clinicians experienced in methadone use; all other HCPs are advised to consult a palliative care specialist. Potency of methadone depends on degree of chronic exposure to morphine. Before initiating methadone, consider morphine dose, duration of dosing, drug interactions and electrocardiogram testing (EKG), especially in patients at risk of prolonged corrected QT (QTC) interval or when doses exceed $30-40 \mathrm{mg} /$ day and again at $100 \mathrm{mg} / \mathrm{day}$. Do not use methadone if QTc $>500 \mathrm{~ms}$; alternate opioids are recommended with QTc $450-500 \mathrm{~ms}$. After initiating methadone, monitor for drug accumulation, QT interval, AEs, especially over first 4-5 days. Perform follow-up EKG in patients at risk of prolonged QTc interval. No evidence-based ratio to convert from methadone to other opioids.

b. Only recommended in opioid-tolerant patients with well-controlled pain. Contraindications: opioid-nailve, unstable pain, increased body temperature due to fever, topical application of heat, or exertion. No evidence-based ratio to convert from transdermal fentanyl to other opioids.

c. An as-needed dose of a short-acting opioid may be necessary during initiation of therapy.

Fig. 3 (continued)

references of the relevant clinical guidelines, case studies and presentation slides, and access to the webcast of the educational session. Dissemination of the toolkit can be supported through the development of a centralized online platform, including an application for mobile devices; such an application may collect data on the usage of the toolkit (e.g., based on the number of downloads and invited structured feedback). Additionally, the online platform would allow HCPs across various specialties (oncology, pain, and palliative care) to share a common confidential forum for clinical case discussions.

\section{Pilot implementation study}

We consider that pilot studies will be essential to demonstrate the feasibility and potential effectiveness of the algorithms and the education program and intend that the selection of sites for pilot studies must consider the involvement of local stakeholders and collaborating partners (government, healthcare system, academic institutions, societies [cancer, pain and palliative care], and industrial partners) and funding sources.

The following aspects are important in designing a pilot implementation study (Fig. 4).

\section{- Baseline data prior to piloting educational program}

Baseline quantitative and qualitative data of the predetermined parameters can be collected through surveys, interviews, or focus groups of HCPs, patients, and policymakers. This step can be facilitated through an online platform (including an application for mobile devices), which can be used for subsequent monitoring and data collection following the educational program.

- Goals and outcomes of the pilot study

Goal 1 (short-term): to improve knowledge and address opiophobia of HCPs

Goal 2 (medium-term): to use the algorithms to improve appropriate use of opioids in CPM

Goal 3 (long-term): to improve broader patient- 


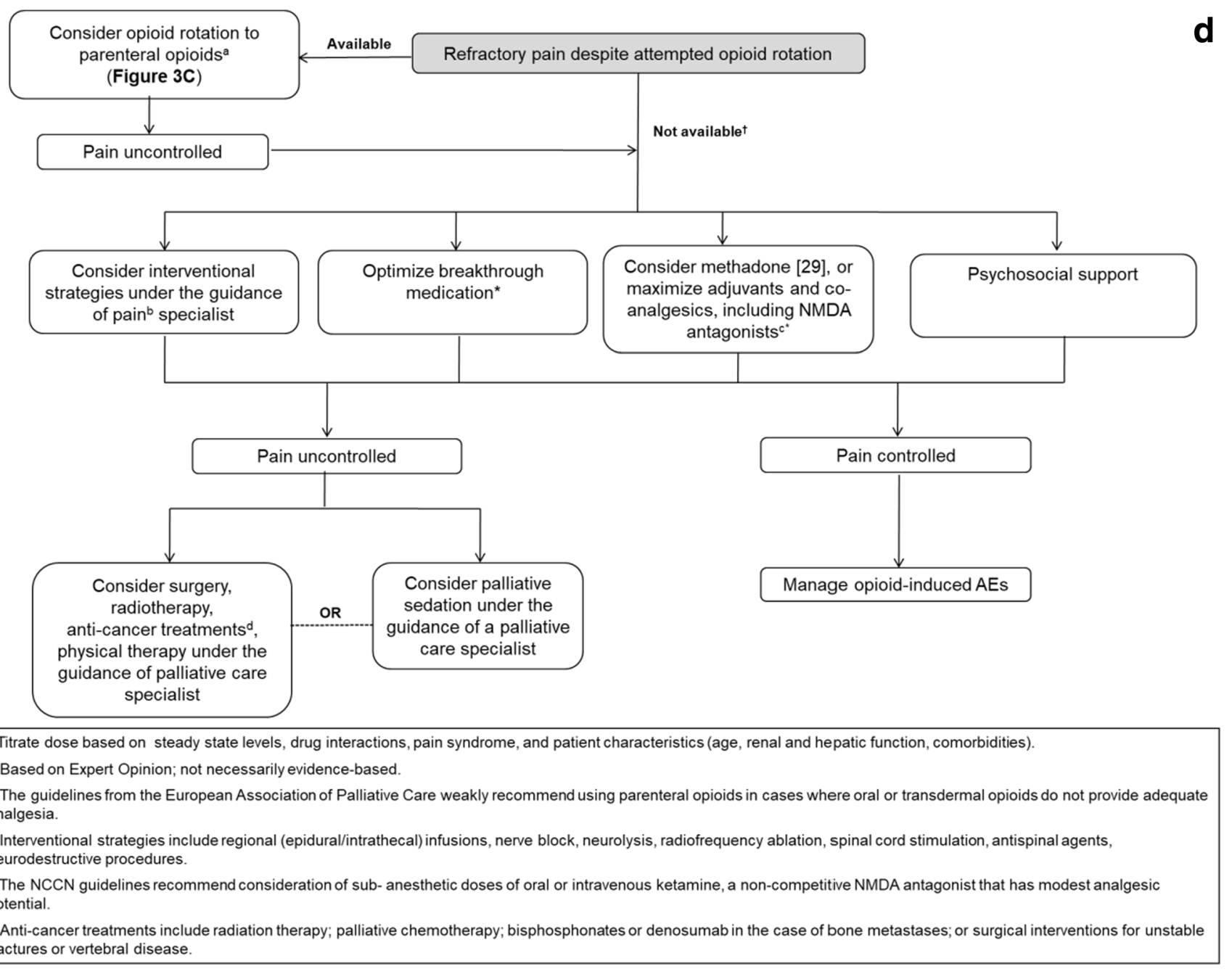

Fig. 3 (continued)

reported outcomes (PROMs) for patients with cancer pain (The desired PROMs include improved pain scores, reduced interference with daily living, increased satisfaction with care and quality of life.)

- Parameters to measure effectiveness of the algorithms and education program

These parameters may vary depending on the country or setting where the program is being piloted, e.g., where opioid availability is a particular issue, where cancer services are under-resourced, or where patient opiophobia is common. They should assess a change in knowledge (e.g., pre- and post-activity test, surveys of intention to put knowledge into practice), a change in attitudes, as well as a change in behavior (e.g., assessing quality indicators such as chart audits, observing HCPs-patients encounter).

- Post-activity monitoring, data collection, and outcomes analysis and research

Ongoing support is required during the pilot studies and later in the actual implementation, to assist participants in the change process. This includes conducting regular discussion sessions, providing regular information updates, and developing online community forums for confidential clinical case discussions and collecting feedback on practical challenges of implementation.

The results from pilot implementation will allow for adjustment/refinement of the strategy, educational program, and the algorithms. Subsequent outcomes research will inform the utility, effectiveness, and sustainability of algorithms and the educational programs.

\section{Advocacy}

Strategic advocacy will be essential to ensure the achievement of CAPER WG program's objective of facilitating highquality care based on adapted evidence-based guidelines to improve CPM in resource-limited settings. We outline the key elements to be included in order to achieve a comprehensive strategic advocacy: 
Table 1 Proposed format and content outline of educational program as part of the framework

Proposed format of educational program:

- Short lectures, interactive case studies discussion, and workshops

- Can be conducted in face-to-face meetings and/or online webcasts

- Toolkit can be provided to participants after completion of the educational program as a continuing educational resource

Content outline of educational program:

- Short lectures

- Basic information, including pathophysiology of pain, pain assessment, determining optimal dose of opioids, opioid rotation, use of adjuvant agents, minimizing adverse events (AEs) caused by the use of opioids, addressing AEs caused by other factors, re-evaluation of dose, and importance of multidisciplinary approach in CPM

- Introduction to the resource-based algorithms

- Interactive case studies discussion to emphasize lecture points and illustrate the practical use of the algorithms for decision-making under resource limitation. For example:

- Case 1: A 40-year old female with upper limb sarcoma had brachial plexus invasion and experienced severe pain that was uncontrolled with $260 \mathrm{mg}$ morphine daily. She was given $40 \mathrm{mg}$ morphine, as needed, as rescue medication, and now experiences spontaneous episodes of pain with myoclonic jerks. What is the cause of this change and what would you do next?

- Case 2: A patient with cancer, whose pain is being managed with regular prolonged-release oxycodone, is currently experiencing breakthrough pain. What would be the treatment of choice if short-acting opioids are not available?

-Workshops for participants to implement the algorithms in various patient scenarios under resource limitation, using a multidisciplinary approach

- An advocacy document (Table 2) that is developed with and endorsed by key international, non-governmental, and professional organizations already active in this area, as well as industrial partners and organizations representing patients.

- Partnerships with international organizations that are already active in improving equity, such as the World Health Organization (WHO), International Association of Hospice and Palliative Care (IAHPC), International Association for the Study of Pain (IASP), and The Union for International Cancer Control (UICC).

- Active engagement with healthcare leaders, local associations/societies across the regions, and senior academics, as well as industrial partners and patient groups, in efforts to improve access.

\section{Discussion}

The Declaration of Montréal, a document developed during the IASP First International Pain Summit on September 3, 2010 , states that access to pain management is a fundamental human right [30]. The Declaration recognized the right of all people to have access to pain management without discrimination, acknowledgement of their pain and to be informed about how it can be assessed and managed, and access to appropriate assessment and treatment of the pain by adequately trained healthcare professionals [30].

Cancer-related pain is a very common cause of persistent pain. While global inequities in CPM have been well-documented, few initiatives or international CPM guidelines, which are generated in high-income countries, specifically consider global variations in resources and access or have provided practical, evidence-based solutions that can be applied to lower-income regions. The CAPER WG proposal endorses the Montreal declaration and is an attempt to address the specific area of cancer-related pain, focusing on patients and HCPs in resource-limited settings.

The WHO analgesic ladder for cancer pain relief [31] has been influential as an educational tool but has not been updated to consider contemporary approaches to pain control and the prevention and management of opioid-induced AEs [32]. While several initiatives have created resource-stratified guidelines for oncology and palliative care [33, 34], we believe that the CAPER WG program is the first to provide resource-sensitive management pathways, based on modern research evidence, for managing cancer pain. Through its two-part initiative described here, the multinational and multidisciplinary CAPER WG has taken on the challenge of bridging the gap between best practice and real-world practice in CPM while accounting for differential resources.

For the first part, we have developed patient-centered and evidence-informed algorithms to provide guidance for the rational management of cancer pain in disparate healthcare settings. These algorithms cover the most important challenges for HCPs in managing cancer pain at all stages: first-line initiation of opioids, breakthrough pain, opioid rotation, and refractory pain. Future work will include the prescribing of other drug groups and non-pharmacological interventions that are important in CPM. The algorithms allow for application of the "best practice" according to "first-world"-derived guidelines, adapted to account for reduced healthcare resources and specifically availability of opioids in resource-limited settings. Our approach in designing these adaptive algorithms was through a rigorous review of existing guidelines and algorithms and ultimately focusing on those produced by NCCN as being the most universally applicable. Members of the CAPER WG from 10 countries, covering both resource-rich and resource-limited settings, provided adaptations which allowed for variations in access, without compromising the clinical aim of optimal CPM.

For the second part, we have recommended a framework to support the implementation of the algorithms to achieve optimal CPM under resource-limited settings. While respecting the important work of international agencies such as the WHO and the International Narcotics Control Board, we recognize that focusing exclusively on overcoming governmental barriers to 
Fig. 4 Outline of pilot implementation plan

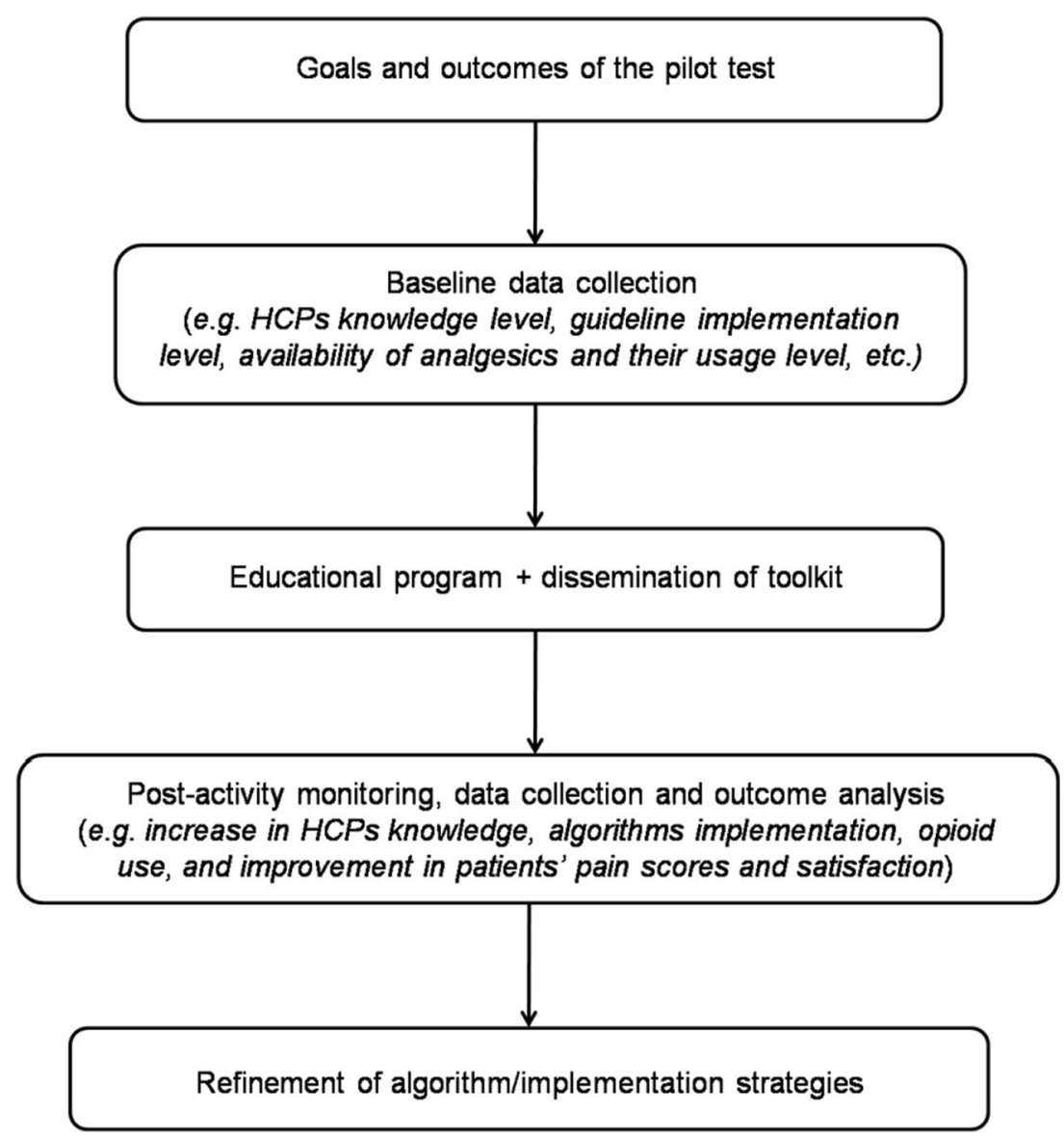

opioid availability has produced only small-scale and geographically patchy successes. Thus, through our practical algorithmic and educational approach, we seek to accelerate improvements in CPM in resource-limited settings by building on the best currently available evidence-based approaches, modified by what is currently available in terms of HCPs, drugs, and other modalities of treatment, ultimately resulting in improved outcomes for patients with cancer.

Furthermore, optimal CPM is impacted by negative perceptions of opioids that often stem from inadequate awareness of pain control and inappropriate use of analgesics among patients and HCPs, who often lack knowledge and confidence in using opioids for cancer pain and managing opioid-related AEs [10, 18, 35-37]; Supplementary resource 7]. These professional barriers are further compounded by poor continuing education, insufficient physician interest, and lack of palliative care education during medical training [7, 19, 20]. Therefore, while our recommendations are designed to facilitate clinical decision-making, we also recognize the need for comprehensive educational and advocacy efforts to overcome sociocultural barriers to effective care.

The effectiveness of educational interventions targeting HCPs to improve cancer pain outcomes has been reviewed; a majority of these interventions were provided in the form of half- or full-day educational meetings involving workshops, lectures, group discussions, and case studies, along with distribution of accompanying print materials [38]. An educational implementation of a cancer pain algorithm for ambulatory care demonstrated that the effect of intervention (a day-long seminar, written reference materials, and documentation tools) was only seen within the first 140 days post-intervention and followed by a gradual decline, highlighting the need for a knowledge retention component [39]. Although educational videotapes and case scenario review on CPM were shown to improve knowledge scores, there was no data regarding their effect on patient outcomes [40]. Thus, these studies did not provide an allencompassing educational intervention that results in improved patient outcomes. While educational efforts initiated by hospitals or institutions can improve knowledge and attitudes of HCPs, these changes may not necessarily be reflected by the changes in practice, highlighting the need for a more directed educational effort that includes practical elements [41]. Our educational program is intended to illustrate how HCPs can still provide optimal pain management for their patients despite working with varied resource constraints/limitations while still following evidence-based international guidelines. These educational activities would use latest online and interactive technologies that can be readily accessed by internet-enabled equipment in all but the most deprived settings. A specific feature of our program will be the development of regional online forums and 
Table 2 Potential elements of advocacy document to improve availability and accessibility of opioids

- Define current gap and its consequence

$\circ$ Opioids are unavailable and inaccessible for CPM in resource-limited settings; lack of education of HCPs renders suboptimal management of pain in patients with cancer

- At least one-third of patient with cancer pain do not receive analgesics proportional to their pain intensity [2]. Furthermore, $75 \%$ of the world population, predominantly in lower- and middle-income countries, has limited access to adequate pain relief [8]

- Set goals and objectives

- To make opioids available in formularies, to reduce regulatory barriers to improve access to opioids for cancer pain, and to improve HCPs' knowledge on optimizing use of available analgesics

- Identify target audience

$\circ$ HCPs and policymakers (regulatory and health authorities)

- Define the action steps to achieve goals and objectives

- Provide evidence to policymakers that opioids are indispensable for the management of cancer pain:

- Demonstration of successful outcome from pilot study of educational program and implementation of algorithms for CPM (e.g., improved HCPs' knowledge, improvement in guidelines implementation by HCPs, improvement in patients' satisfaction)

- Evidence for patients with cancer whose pain remains uncontrolled despite optimizing available analgesics according to evidence-based guideline recommendations

- Demonstration of safe opioid use for CPM

- Initiate local and regional research projects to collect more sophisticated data on barriers, facilitators, and outcomes of the program

$\circ$ Initiate a national forum for dialogue on improving opioid availability and accessibility

- Define the expected outcomes

- Scaling up of the pilot program, and improvement in HCPs' knowledge and appropriate use of analgesics

- Improvement in the availability of opioids in the formulary

$\circ$ Easing up of regulatory policies to allow better access to opioids

other platforms for continuous updating of knowledge and sharing of experiences, including anonymized case studies.

The CAPER WG pilot studies will serve as an important process to monitor, assess, and evaluate for key patient outcomes and how they translate into a change in healthcare practice that benefit HCPs, patients, and potentially the healthcare organization/system.

We acknowledge successful collaborative efforts to improve availability and access to opioids, involving cooperation from the national governments and regulatory authorities, have been demonstrated in Vietnam, Nepal, Serbia, and subSaharan Africa. Vietnam and Serbia have seen an increased in opioid consumption [42, 43], while Nepal now manufactures three forms of oral morphine locally [44] and Uganda has seen an increase in morphine availability [45]. These examples highlight the importance of continuous collaborative and multifaceted efforts involving committed stakeholders including industry, led by local champions.

\section{Limitations of the CAPER WG program and their solutions}

The CAPER WG is a consortium of interested parties who have a long experience of researching and applying evidence-based guidelines for CPM in both resource-rich and resource-limited regions. The main geographical areas covered by the WG are in the Far East and Asia-Pacific regions, but also with representation from Middle East/North Africa, Latin America, and the UK. The consortium thus represents a very broad multiprofessional and global perspective on CPM. It is not a legal entity but will seek endorsement from existing international and regional/national organizations once the project has yielded tangible results. Additional perspectives from other regions, notably sub-Saharan Africa, Indian subcontinent, Eastern Europe and the former Baltic States, and Russia could enhance the program once it is underway.

The algorithms we have adapted have not been formally validated, but this is consistent with many of the international guidelines which are currently employed. However, we have focused on the NCCN guidelines which are evidence-based. Furthermore, our CAPER WG implementation program has a component that will prospectively collect data on usability and challenges and, importantly, will measure outcomes relevant to patients with cancer.

The success of the implementation of the algorithms and the educational programs may be influenced and hampered by various factors related to HCPs, policymakers, and patients. HCPs may be discouraged from implementing changes in their clinical practice because of fear of isolation by peers, lack of time or resources, or non-supportive healthcare systems (e.g., lack of organizational support). Policymakers may be reluctant or disinterested owing to lack of understanding of the ground reality or having different priority agenda. Patients may have persistent fears associated with opioid use. Additionally, industry-led initiatives are often viewed by HCPs and policymakers with suspicion. These barriers can potentially be overcome by generating local clinician engagement, academic support through research activities, and broad-based organizational advocacy.

The CAPER WG feels that after decades of the initial groundbreaking $1986 \mathrm{WHO}$ cancer pain program, it is time for a bold new initiative to take on the cause of bringing evidence-based CPM to resource-limited parts of the world. We look forward to collaborating with both pain and cancer global and regional organizations in coming years.

\section{Conclusion}

The CAPER WG has taken the first two steps to correct the imbalance of providing optimal CPM in resource-limited settings. We have proposed a practical and implementable strategy that addresses the challenges in translation of best practices, 
owing to resource constraints and/or lack of education and awareness. First, the four algorithms cover the key areas that prevent safe and appropriate use of opioids in different clinical scenarios. Second, the education and implementation framework is an essential move to disseminate the algorithms and test their usability and clinical outcomes. Integral to implementation is an advocacy program, to influence key stakeholders, non-governmental organizations, and industry for the advancement of CPM in resource-limited settings. We invite further discussion of the algorithms and extend an invitation to participants in the education and implementation framework.

Acknowledgements Editorial assistance for this manuscript was provided by Yulyana and Aishwarya Sridharan from In Vivo Communications, funded by Mundipharma Pte Ltd.

Funding information This work was supported by Mundipharma Pte Ltd.

\section{Compliance with ethical standards}

Conflict of interest MJB, YG, AAIH, SH, DK, DCK, DHL, and JJW received sponsorship from Mundipharma for attending the steering committee meeting.

SHA has received payments for conducting research, giving educational lectures, and taking part in advisory boards with Astra Zeneca, Grünenthal, Mundipharma, and Pfizer; for conducting research with Kyowa Kirin; and for giving educational lectures for Menarini. MJB has received remunerations for lectures given for Johnson and Johnson (formerly Janssen Pharmaceuticals) and Fondazione Menarini. RG has received payments for conducting research, giving educational lectures, and taking part in advisory boards with Onyx, Kaleido Biosciences, and Mundipharma. SH has received lecture fees for Shionogi Pharmaceuticals Co., Mundipharma Japan Co., Taiho Pharmaceutical Co., Daiichi Sankyo Co., Hisamitsu Co., and Teikoku Pharmaceutical Co. DK serves on the Advisory Board of Entrinsic Health Solutions. DCK serves on the Latin America Board of the Grünenthal Group and received remunerations for lectures for Cristalia Produtos Químicos e Farmacêuticos. DHL has received honoraria from AstraZeneca, Boehringer-Ingelheim, BristolMyers Squibb, CJ Healthcare, Eli Lilly, Janssen, Merck, MSD, Mundipharma, Novartis, Ono, Pfizer, Roche, Samyang Biopharm, ST Cube, AbbVie, and Takeda for participating in advisory boards. KT has received remunerations for lectures from Kyowa Hakko Kirin Co. Ltd., Ono Pharmaceutical Co., and Eisai Co. JJW has received remunerations for lectures from Eli Lilly, Roche, and Mundipharma. KB, YG, and AAIH have no other conflicts of interest to declare.

Open Access This article is distributed under the terms of the Creative Commons Attribution-NonCommercial 4.0 International License (http:// creativecommons.org/licenses/by-nc/4.0/), which permits any noncommercial use, distribution, and reproduction in any medium, provided you give appropriate credit to the original author(s) and the source, provide a link to the Creative Commons license, and indicate if changes were made.

\section{References}

1. van den Beuken-van Everdingen MH, Hochstenbach LM, Joosten EA et al (2016) Update on prevalence of pain in patients with cancer: systematic review and meta-analysis. J Pain Symptom Manag 51:1070-1090
2. Greco MT, Roberto A, Corli O, Deandrea S, Bandieri E, Cavuto S, Apolone G (2014) Quality of cancer pain management: an update of a systematic review of undertreatment of patients with cancer. J Clin Oncol 32(36):4149-4154

3. Paice JA, Von Roenn JH (2014) Under- or overtreatment of pain in the patient with cancer: how to achieve proper balance. J Clin Oncol 32(16): 1721-1726

4. Van Riet Paap J, Vissers K, Iliffe S, Radbruch L, Hjermstad MJ, Chattat R, Vernooij-Dassen M, Engels Y, research team IMPACT (2015) Strategies to implement evidence into practice to improve palliative care: recommendations of a nominal group approach with expert opinion leaders. BMC Palliat Care 14:47

5. World Health Organization and Worldwide Palliative Care Alliance (2014) Global atlas of palliative care at the end of life. http://www. who.int/nmh/Global_Atlas_of_Palliative_Care.pdf. Accessed 6 November 2017

6. Hastie BA, Gilson AM, Maurer MA, Cleary JF (2014) An examination of global and regional opioid consumption trends 1980 2011. J Pain Palliat Care Pharmacother 28:259-275

7. Human Rights Watch (2011) Global state of pain treatment: access to palliative care as a human right. https://www.hrw.org/sites/ default/files/reports/hhr0511W.pdf. Accessed 7 November 2017

8. International Narcotics Control Board (2015) Availability of internationally controlled drugs: ensuring adequate access for medical and scientific purposes, indispensable, adequately available and not unduly restricted. https://www.incb.org/documents/Publications/ AnnualReports/AR2015/English/Supplement-AR15_availability_ English.pdf. Accessed 6 November 2017

9. Cleary J, Radbruch L, Torode J, Cherny NI (2013) Formulary availability and regulatory barriers to accessibility of opioids for cancer pain in Asia: a report from the Global Opioid Policy Initiative (GOPI). Ann Oncol 24(Suppl 11):xi24-xi32

10. Javier F, Irawan C, Mansor M, Sriraj W, Tan KH, Thinh DHQ (2016) Cancer pain management insights and reality in Southeast Asia: expert perspectives from six countries. J Global Oncology 2:235-243

11. Berterame S, Erthal J, Thomas J, Fellner S, Vosse B, Clare P, Hao W, Johnson DT, Mohar A, Pavadia J, Samak AKE, Sipp W, Sumyai V, Suryawati S, Toufiq J, Yans R, Mattick RP (2016) Use of and barriers to access to opioid analgesics: a worldwide, regional, and national study. Lancet 387:1644-1656

12. Cleary J, De Lima L, Eisenchlas J, Radbruch L, Torode J, Cherny NI (2013) Formulary availability and regulatory barriers to accessibility of opioids for cancer pain in Latin America and the Caribbean: a report from the Global Opioid Policy Initiative (GOPI). Ann Oncol 24(Suppl 11):xi41-xi50

13. Cleary J, Silbermann M, Scholten W, Radbruch L, Torode J, Cherny NI (2013) Formulary availability and regulatory barriers to accessibility of opioids for cancer pain in the Middle East: a report from the Global Opioid Policy Initiative (GOPI). Ann Oncol 24(Suppl 11):xi51-xi59

14. Kerr S, Jazieh AR, Kerr D (2017) How useful are international treatment guidelines in low- and middle-income countries? J Glob Oncol 3(5):441-443

15. Vander Schaaf EB, Seashore CJ, Randolph GD (2015) Translating clinical guidelines into practice: challenges and opportunities in a dynamic health care environment. N C Med J 76(4):230-234

16. Baiardini I, Braido F, Bonini M, Compalati E, Canonica GW (2009) Why do doctors and patients not follow guidelines? Curr Opin Allergy Clin Immunol 9:228-233

17. Fischer F, Lange K, Klose K, Greiner W, Kraemer A (2016) Barriers and strategies in guideline implementation - a scoping review. Healthcare (Basel) 4(3):pii:E36

18. ACHEON Working Group, Kim YC, Ahn JS, Calimag MM, Chao TC, Ho KY, Tho LM, Xia ZJ, Ward L, Moon H, Bhagat A (2015) Current practices in cancer pain management in Asia: a survey of 
patients and physicians across 10 countries. Cancer Med 4(8): 1196-1204

19. Pastrana T, Centeno C, De Lima L (2015) Palliative care in Latin America from the professional perspective: a SWOT analysis. J Palliat Med 18(5):429-437

20. Silbermann M, Arnaout M, Daher M, Nestoros S, Pitsillides B, Charalambous H, Gultekin M, Fahmi R, Mostafa KA, Khleif AD, Manasrah N, Oberman A (2012) Palliative cancer care in Middle Eastern countries: accomplishments and challenges. Ann Oncol 23(Suppl 3):15-28

21. Cleary J, Powell RA, Munene G, Mwangi-Powell FN, Luyirika E, Kiyange F, Merriman A, Scholten W, Radbruch L, Torode J, Cherny NI (2013a) Formulary availability and regulatory barriers to accessibility of opioids for cancer pain in Africa: a report from the Global Opioid Policy Initiative (GOPI). Ann Oncol 24(Suppl 11):xi14-xi23

22. Cleary J, Simha N, Panieri A, Scholten W, Radbruch L, Torode J, Cherny NI (2013c) Formulary availability and regulatory barriers to accessibility of opioids for cancer pain in India: a report from the Global Opioid Policy Initiative (GOPI). Ann Oncol 24(Suppl 11): xi33-xi40

23. Morriss W, Goucke R (2016) Essential pain management workshop manual, 2nd edn. http://fpm.anzca.edu.au/documents/epmparticipant-manual-082016.pdf. Accessed 7 November 2017

24. Yamamoto R, Kizawa Y, Nakazawa Y, Ohde S, Tetsumi S, Miyashita M (2015) Outcome evaluation of the palliative care emphasis program on symptom management and assessment for continuous medical education: nationwide physician education project for primary palliative care in Japan. J Palliat Med 18(1):45-49

25. NCCN, Clinical Practice Guidelines in Oncology. Adult Cancer Pain Version 2 (2016) National Comprehensive Cancer Network. https://www.nccn.org/professionals/physician_gls/default.aspx. Accessed 7 November 2017

26. Chou R, Gordon DB, de Leon-Casasola OA et al (2016) Management of postoperative pain: a clinical practice guideline from the American Pain Society, the American Society of Regional Anesthesia and Pain Medicine, and the American Society of Anesthesiologists' Committee on Regional Anesthesia, Executive Committee, and Administrative Council. J Pain 17:131157

27. Davies AN, Dickman A, Reid C, Stevens AM, Zepetella G (2009) The management of cancer-related breakthrough pain: recommendations of a task group of the Science Committee of the Association for Palliative Medicine of Great Britain and Ireland. Eur J Pain 13: 331-338

28. Caraceni A, Hanks G, Kaasa S, Bennett MI, Brunelli C, Cherny N, Dale O, de Conno F, Fallon M, Hanna M, Haugen DF, Juhl G, King S, Klepstad P, Laugsand EA, Maltoni M, Mercadante S, Nabal M, Pigni A, Radbruch L, Reid C, Sjogren P, Stone PC, Tassinari D, Zeppetella G, European Palliative Care Research Collaborative (EPCRC)., European Association for Palliative Care (EAPC) (2012) Use of opioid analgesics in the treatment of cancer pain: evidence-based recommendations from the EAPC. Lancet Oncol 13:e58-e68

29. Afsharimani B, Kindl K, Good P, Hardy J (2015) Pharmacological options for the management of refractory cancer pain - what is the evidence? Support Care Cancer 23:1473-1481

30. International Association for the Study of Pain (2010) Declaration of Montréal. https://www.iasp-pain.org/DeclarationofMontreal. Accessed 15 January 2018
31. World Health Organization (1996) Cancer pain relief with a guide to opioid availability, 2nd edn. http://apps.who.int/iris/bitstream/ 10665/37896/1/9241544821.pdf. Accessed 7 November 2017

32. Ahmedzai SH (2014) Cancer pain for the 21st century: stepping off the ladder, stepping up to new challenges. Br J Pain 8:131-132

33. NCCN, NCCN Framework for Resource Stratification of NCCN Guidelines (NCCN Framework ${ }^{\mathrm{TM}}$ ). National Comprehensive Cancer Network. https://www.nccn.org/framework/default.aspx. Accessed 7 November 2017

34. Payne S, Chan N, Davies A, Poon E, Connor S, Goh C (2012) Supportive, palliative, and end-of-life care for patients with cancer in Asia: resource-stratified guidelines from the Asian Oncology Summit 2012. Lancet Oncol 13:e492-e500

35. Chen C, Tang S, Chen C (2012) Meta-analysis of cultural differences in Western and Asian patient-perceived barriers to managing cancer pain. Palliat Med 26:206-221

36. Yamaguchi T, Narita M, Morita T, Kizawa Y, Matoba M (2012) Recent developments in the management of cancer pain in Japan: education, clinical guidelines and basic research. Jpn J Clin Oncol 42:1120-1127

37. Peacock A, Nielsen S, Bruno R, Campbell G, Larance B, Degenhardt L (2016) Geographic variation in health service use and perceived access barriers for Australian adults with chronic non-cancer pain receiving opioid therapy. Pain Med 17:2003-2016

38. Cummings GG, Olivo SA, Biondo PD, Stiles CR, Yurtseven O, Fainsinger RL, Hagen NA (2011) Effectiveness of knowledge translation interventions to improve cancer pain management. J Pain Symptom Manag 41(5):915-939

39. Du Pen AR, Du Pen S, Hansberry J, Miller-Kraybill B, Millen KJ, Everly R et al (2000) An educational implementation of a cancer pain algorithm for ambulatory care. Pain Manag Nurs 1(4):116-128

40. Patiraki EI, Papathanassoglou ED, Tafas C, Akarepi V, Katsaragakis SG, Kampitsi A et al (2006) A randomized controlled trial of an educational intervention on Hellenic nursing staff's knowledge and attitudes on cancer pain management. Eur $\mathrm{J}$ Oncol Nurs 10(5):337-352

41. Kasasbeh MAM, McCabe C, Payne S (2017) Cancer-related pain management: a review of knowledge and attitudes of healthcare professionals. Eur J Cancer Care (Engl) 26(6):1-7

42. Krakauer EL, Nguyen TP, Husain SA, Nguyen TH, Joranson DE, Luong NK, Maurer MA (2015) Toward safe accessibility of opioid pain medicines in Vietnam and other developing countries: a balanced policy method. J Pain Symptom Manag 49(5):916-922

43. Bosnjak SM, Maurer MA, Ryan KM, Popovic I, Husain SA, Cleary JF, Scholten W (2016) A multifaceted approach to improve the availability and accessibility of opioids for the treatment of cancer pain in Serbia: results from the International Pain Policy Fellowship (2006-2012) and recommendations for action. J Pain Symptom Manag 52(2):272-283

44. Paudel BD, Ryan KM, Brown MS, Krakauer EL, Rajagopal MR, Maurer MA, Cleary JF (2015) Opioid availability and palliative care in Nepal: influence of an international pain policy fellowship. J Pain Symptom Manag 49(1):110-116

45. O'Brien M, Mwangi-Powell F, Adewole IF, Soyanwo O, Amandua J, Ogaja E, Okpeseyi M, Ali Z, Kiwanuka R, Merriman A (2013) Improving access to analgesic drugs for patients with cancer in subSaharan Africa. Lancet Oncol 14(4):e176-e182 